\title{
Layered Video Transmission on Adaptive OFDM Wireless Systems
}

\author{
D. Dardari \\ IEIIT-BO/CNR, CNIT, DEIS, University of Bologna, Viale Risorgimento 2, 40136 Bologna, Italy \\ Email:ddardari@deis.unibo.it
}

M. G. Martini

IEIIT-BO/CNR, CNIT, DEIS, University of Bologna, Viale Risorgimento 2, 40136 Bologna, Italy

Email: mgmartini@deis.unibo.it

M. Mazzotti

IEIIT-BO/CNR, CNIT, DEIS, University of Bologna, Viale Risorgimento 2, 40136 Bologna, Italy Email:mmazzotti@deis.unibo.it

\author{
M. Chiani \\ IEIIT-BO/CNR, CNIT, DEIS, University of Bologna, Viale Risorgimento 2, 40136 Bologna, Italy \\ Email:mchiani@deis.unibo.it
}

Received 28 February 2003; Revised 26 January 2004

\begin{abstract}
Future wireless video transmission systems will consider orthogonal frequency division multiplexing (OFDM) as the basic modulation technique due to its robustness and low complexity implementation in the presence of frequency-selective channels. Recently, adaptive bit loading techniques have been applied to OFDM showing good performance gains in cable transmission systems. In this paper a multilayer bit loading technique, based on the so called "ordered subcarrier selection algorithm," is proposed and applied to a Hiperlan2-like wireless system at $5 \mathrm{GHz}$ for efficient layered multimedia transmission. Different schemes realizing unequal error protection both at coding and modulation levels are compared. The strong impact of this technique in terms of video quality is evaluated for MPEG-4 video transmission.
\end{abstract}

Keywords and phrases: OFDM, adaptive modulation, bit loading, UEP, MPEG-4.

\section{INTRODUCTION}

One of the main goals in the near future of communication systems is the development of multimedia efficient data coding, compression, and transmission techniques that permit real-time mobile communications. In this context, the major challenge is the integration of different categories of networks and wireless local area networks (WLAN). Systems have to be adaptive, that is, they have to react to changing quality conditions, like varying channel capacity.

In high-speed wireless data applications, the orthogonal frequency division multiplexing (OFDM) modulation scheme has been considered due to its relatively simple receiver structure compared to single-carrier transmission in frequency-selective fading channels. OFDM modulation is adopted by IEEE for the extension of the 802.11 wireless LAN standard to the $5 \mathrm{GHz}$ band (IEEE802.11a), providing data rates up to $54 \mathrm{Mbps}$ [1]. ETSI adopted the OFDM scheme for the high performance LAN physical layer standard (Hiperlan2) As well [2].

Conventional OFDM modems use fixed constellation size and power level allocation of all subchannels. In more recent standards (i.e., IEEE802.11a), the adaptation of the constellation size (the same for all subchannels) according to the global channel-state time-variation is admitted. Due to multipath fading, some subchannels could experience severe degradation in the signal-to-noise ratio (SNR), resulting in high overall bit error rates. Channel coding is a common technique to mitigate this effect. If the channel is static (e.g., in digital subscribers lines (DSL)) or slowly time varying, the receiver can provide the transmitter with detailed channel state information (CSI) using a robust feedback channel. Based on the CSI, more sophisticated adaptive transmission techniques have the possibility to dynamically modify 
the parameters of the modulator in order to improve the performance [3]. Thanks to the characteristic of multicarrier modulations, it is also possible to dynamically change the transmitting power and bit rate of each subchannel according to channel selectivity variations (adaptive bit loading).

The first applications of bit loading algorithms appeared in DSL systems $[4,5]$. It is a well-known fact that the theoretical channel capacity can be approached by distributing the total transmitted energy according to the water-filling principle [6]. In the realistic case where a finite granularity in constellation size is required, the rounded bit distribution obtained starting from the water-filling solution could still not be the optimum. Some suboptimum algorithms to reduce the complexity have been proposed in the ADSL context [7, 8]. Campello [9] gives the theoretically sufficient conditions for a discrete bit allocation to be optimal. Based on his conditions, a "greedy" algorithm can be used to achieve the optimal discrete bit/power loading distribution.

Recently, some studies regarding the application of adaptive bit loading algorithms to wireless channels appeared $[10,11,12,13]$. In this case, particular attention must be paid to channel estimation and CSI update rate effects on the performance $[14,15,16]$. However, water-filling-based techniques require a large overhead for CSI feedback, making them suitable only for static or very slow time varying channels. Moreover, the modem must be able to continually change the modulation format and power on subcarrier basis (high complexity if high data rates are requested). Hence, simple suboptimal algorithms should be investigated in order to reduce complexity and CSI overhead.

The adaptation of the modulation segment also to the source data structure and significance may provide good results by realizing unequal error protection (UEP) in the modulation domain. UEP has proven to provide good performance in the case of transmission of compressed sources, where the bits produced have a different significance. Providing a lower bit error rate for the bits with higher significance and leaving the less significant bits with less protection makes it possible to increase the perceived quality. UEP has been applied for audio transmission $[17,18]$, for progressive image transmission [19], and for subband coded audio and video transmission, as some kinds of sources lend themselves to be partitioned into differently sensitive groups of bits. Also UEP for block-based video coded sources has been proposed as in $[20,21,22]$.

UEP is classically performed at channel coding level, through convolutional and, more recently, turbo codes. Multiresolution constellations allows a nonuniform data protection in the modulation domain [23]. Some recent studies have proposed to perform UEP in the modulation domain, exploiting the characteristics of multicarrier modulations $[13,24]$. In this case, the fact that a nonuniform bit and power allocation among the subcarriers is required implies a significant modem complexity and a high CSI signaling overhead between the transmitter and the receiver with respect to the uniform case. This may cause a higher sensitivity to signaling errors.
In this paper, a simple bit loading algorithm, where the constellation size and power levels are constrained to be uniform for all used subcarriers, is proposed and extended to the multilayer case to perform UEP of layered video sources at the modulation level. This technique is compared with UEP realized at channel coding level and with an equal error protection (EEP) scheme based on classic bit loading techniques. The performance evaluation in terms of peak signal-to-noise ratio (PSNR) for MPEG-4 video transmission for wireless data service is addressed, showing the large gain that can be obtained, especially at low SNRs.

\section{REFERENCE TRANSMISSION SYSTEM}

Figure 1 illustrates the considered transmission system. The transmitter section is basically made up of a channel coder followed by a bit loading unit that distributes the data bit, according to the algorithm implemented, among the subchannels (more details about its functions will be given further) and a conventional OFDM modulator. The OFDM scheme allows the transmission of $N$ parallel complex symbols $A_{n}$ $(n=1,2, \ldots, N)$, that belong to an $M_{n}$ points constellation set $\left\{ \pm 1, \pm 3, \ldots, \pm\left(\sqrt{M_{n}}-1\right)\right\}$ for both real and imaginary dimensions, into $N$ parallel subchannels (or subcarriers). The symbol (or frame) duration is denoted with $T_{s}$. Generally, only a limited, and constant over the subcarriers, set of values for $M_{n}=M$ is adopted in practical modems (e.g., $M=2,4,16,64$ ) [1]. In order to grant the orthogonality between subchannels in ideal channel conditions, the subchannel subdivision is obtained by means of an inverse fast Fourier transform (IFFT) of order $N_{\text {FFT }}\left(N<N_{\text {FFT }}\right.$ to accommodate virtual subcarriers). Samples at the output of the IFFT block are converted from parallel to serial and transmitted every $T_{c}$ seconds (chip time). In practice, due to propagation effects, subchannels still do not remain orthogonal, so a cyclic prefix (guard interval) is added to the OFDM symbol (the IFFT output) in order to remove the intersymbol interference (ISI) among subchannels [25]. Its duration is a multiple $D$ of the chip time $T_{c}$, that is, $T_{g}=D \cdot T_{c}$. At the receiver side, the reverse process is performed. The cyclic prefix represents a redundancy, in fact, only the time $T_{u}=N_{\mathrm{FFT}} \cdot T_{c}$ is dedicated to the transmission of useful data, whereas the total OFDM symbol time is $T_{s}=T_{u}+T_{g}=T_{c} \cdot\left(N_{\mathrm{FFT}}+D\right)$. The power efficiency (less than 1 ) due to the guard interval is

$$
\eta_{D}=\frac{T_{u}}{T_{s}}=\frac{N_{\mathrm{FFT}}}{\left(N_{\mathrm{FFT}}+D\right)} .
$$

If the maximum multipath delay $T_{d}$ is less than the guard interval $T_{g}$, no ISI is present and the complex received signal at the $n$th output of the FFT block can be written, in a normalized form, as [26]

$$
Z_{n}=H_{n} \cdot w_{n} \cdot A_{n}+x_{n}
$$

where $H_{n}$ is the channel transfer function gain related to the $n$th subchannel, and $w_{n}$ is a weight coefficient which allows nonuniform power level allocation on the transmitter side as 


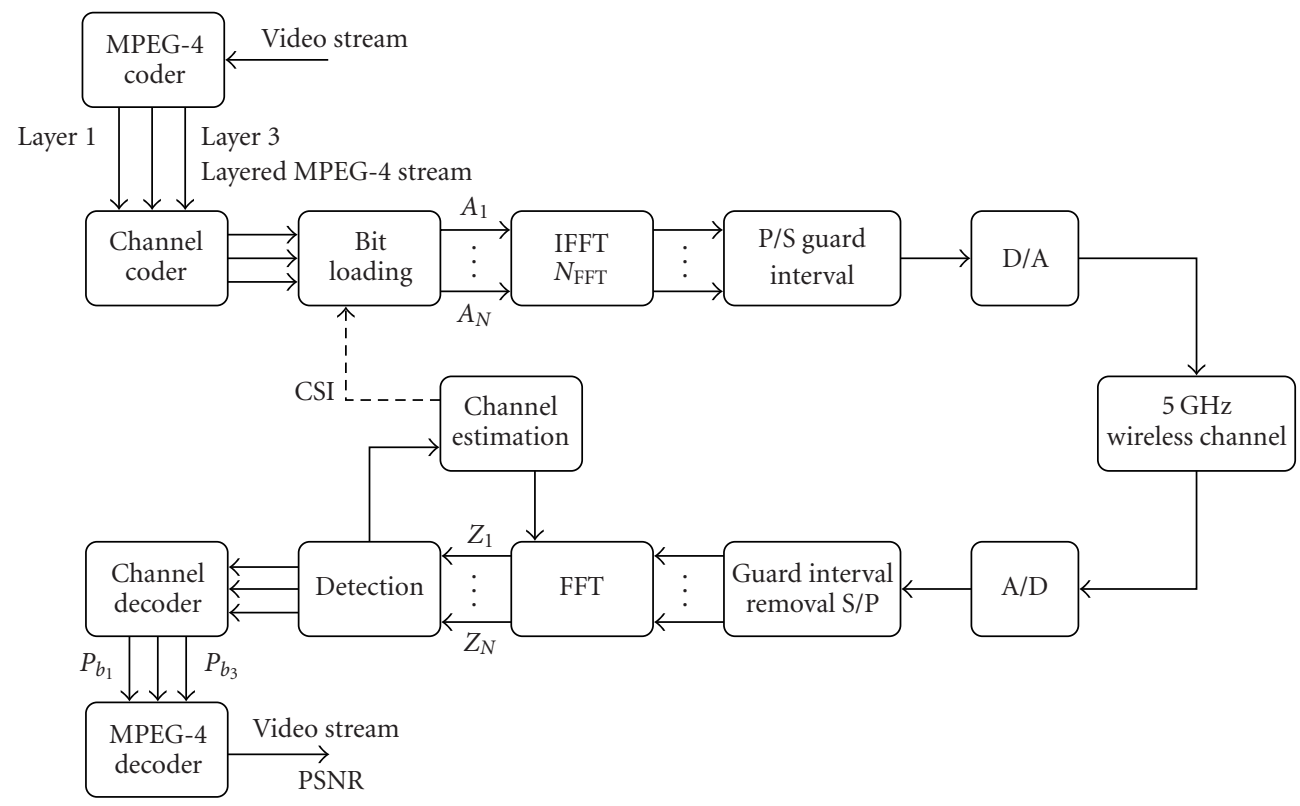

Figure 1: Transmission system block diagram.

required by common bit loading algorithms. In not adapted schemes, $w_{n}=1$ for all $n$. Following the same normalization done in [26], the random variable $x_{n}$ represents the zeromean complex Gaussian thermal noise component at the $n$th FFT output with power

$$
\sigma_{x}^{2}=E\left[\left|x_{n}\right|^{2}\right]=\frac{2 N_{0}}{T_{u}},
$$

where $N_{0}$ is the single-side power noise density. Recalling that symbol $A_{n}$ belongs to an $M_{n}$-QAM constellation, the average power $P_{n}$ dedicated to the $n$th subchannel is

$$
P_{n}=E\left[\left|A_{n}\right|^{2}\right] \cdot w_{n}^{2}=\frac{2\left(M_{n}-1\right) w_{n}^{2}}{3},
$$

leading to a total average transmitted power $P_{T}$ :

$$
P_{T}=\sum_{n=1}^{N_{u}} P_{n}
$$

$N_{u}$ is the actual number of subchannels used by the bit loading algorithm. We have neglected the presence of pilot subcarriers allocated for channel estimation purposes.

In the case where an $M_{n}$-QAM signaling is adopted, assuming ideal phase offset compensation, perfect carrier recovery and synchronization, the bit error probability related to the $n$th subchannel can be approximated as follows [27]:

$$
P_{b_{n}} \cong \frac{2\left(\sqrt{M_{n}}-1\right)}{\sqrt{M_{n}} \cdot \log _{2} M_{n}} \cdot \operatorname{erfc} \sqrt{\frac{w_{n}^{2} \cdot\left|H_{n}\right|^{2}}{\sigma_{x}^{2}}} .
$$

Considering (3), (4), and (5), we obtain

$$
\begin{aligned}
P_{b_{n}} & \cong \frac{2\left(\sqrt{M_{n}}-1\right)}{\sqrt{M_{n}} \cdot \log _{2} M_{n}} \cdot \operatorname{erfc} \sqrt{\frac{P_{n} \cdot 3\left|H_{n}\right|^{2}}{2\left(M_{n}-1\right) \sigma_{x}^{2}}} \\
& =\frac{2\left(\sqrt{M_{n}}-1\right)}{\sqrt{M_{n}} \cdot \log _{2} M_{n}} \cdot \operatorname{erfc} \sqrt{\frac{E_{s}}{N_{0}} \frac{3 \varepsilon_{n} \cdot\left|H_{n}\right|^{2} \cdot \eta_{D}}{2\left(M_{n}-1\right)}}
\end{aligned}
$$

where

$$
\frac{E_{s}}{N_{0}}=\frac{P_{T} \cdot T_{s}}{2 N_{0}}
$$

denotes the transmitted (OFDM) radio frequency symbol energy-to-noise ratio, and $\varepsilon_{n}=P_{n} / P_{T}$ indicates the fraction of the power dedicated to the $n$th subchannel. Obviously, it is $\sum_{n=1}^{N_{u}} \varepsilon_{n}=1$. Once the code rate, $R_{c}$, and the actual number of bit transmitted per frame, $b_{T}$, are fixed, $E_{s} / N_{0}$ can be expressed as a function of the received average bit energy-tonoise ratio $E_{b} / N_{0}$ :

$$
\frac{E_{s}}{N_{0}}=\frac{E_{b}}{N_{0}} R_{c} b_{T}
$$

As can be noted, the performance at each subchannel depends on $\left|H_{n}\right|$, so severely attenuated subchannels could compromise the performance. In general, a suitable channel coding is necessary to improve the overall performance (coded OFDM) [26] as done in the numerical results. 


\section{MULTILAYER ADAPTIVE BIT LOADING}

\subsection{Ordered subcarrier selection algorithm}

Current WLAN standards $[1,2]$ consider a fixed bit loading scheme where, once the decision on the constellation size $M$ based on overall propagation conditions has been made, all subchannels $\left(N_{u}=N\right)$ utilize the same size $M\left(M_{n}=M\right)$ and the same power fraction $\left(\varepsilon_{n}=1 / N\right)$, independently by the single subchannel condition. In the following, this case is referred to as the reference scheme (conventional OFDM scheme with no adaptation). The total number of bits transmitted by every OFDM symbol time $T_{s}$ is $b_{T}=N \log _{2}(M)$.

The basic principle of adaptive modulation techniques is the opportunity of dynamically modifying the modulation parameters $\left(M_{n}, \varepsilon_{n}\right.$, and $\left.N_{u}\right)$ according to the time-variant channel conditions [3]. This can be accomplished efficiently if the transmitter knows the channel state. A feedback channel should thus be available, as shown in Figure 1, in order to pass the CSI to the transmitter. The rate of CSI depends on the channel variability, in particular on the channel coherence time. Common adaptive schemes require that each subchannel be loaded using a particular constellation size $M_{n}$ and fractional power level $\varepsilon_{n}$, different from that allocated in the other subchannels [7]. The optimal set for $\varepsilon_{n}$ and $M_{n}$, that maximizes the power margin, is given by the Campello's conditions [9]. In those cases, all source bits are assumed to have the same importance (EEP).

These algorithms lead to a high level of modem complexity and the necessity to provide a large signaling overhead in time-varying wireless channels, especially in high-speed systems. To partially overcome this problem, some techniques appeared in the Literature; Grunheid et al. [28] propose a simplified scheme where the optimization is performed with a blockwise allocation of modulation levels. In [29], it is shown that a constant power allocation scheme has a negligible performance loss compared to the optimal solution.

In order to obtain low complexity modems, we herein propose a modified scheme transmitting the same amount of bits $b_{T}$ as in the reference scheme, but where only a subset $N_{u} \leq N$ of the available $N$ subchannels is effectively used. Now, the actual constellation size has to be suitably increased in order to allocate all the $b_{T}$ bits, that is,

$$
M_{n}=M=2^{b_{T} / N_{u}}, \quad n=1,2, \ldots, N_{u} .
$$

Obviously, only a limited number of values for $N_{u}$ is allowed if we want the constellation size $M$ to result in a practical integer value. The total transmitted power is uniformly distributed among the $N_{u}$ used subchannels as a consequence $\left(\varepsilon_{n}=1 / N_{u}\right)$.

The basic idea of the ordered subcarrier selection algorithm that we propose herein is to select only the strongest $N_{u}$ subchannels (i.e., the subchannels characterized by a higher value for $\left|H_{n}\right|^{2}$ ) and to use higher constellation sizes by keeping the total bit rate and transmitted power unchanged. In our approach, both the power level and the constellation size are kept constant over the selected set of subchannels. The receiver's task is to estimate the channel gain $H_{n}$, select the
$N_{u}$ strongest (most reliable) subchannels and, through the feedback channel, inform the transmitter which to use in the next packet transmission. It is to be pointed out that the feedback throughput required is very limited compared to that required by common bit loading algorithms [5].

To find which choice for $N_{u}$ gives good results, we analyze the average bit error probability, obtained by the algorithm proposed, in the case where all subchannels are affected by independent Rayleigh fading. This means that $\lambda=\left|H_{n}\right|^{2}$ is an exponentially distributed random variable [27]. The functions

$$
\begin{gathered}
f_{\lambda}(\lambda)=e^{-\lambda}, \\
F_{\lambda}(\lambda)=1-e^{-\lambda},
\end{gathered}
$$

are the probability density function (pdf) and the cumulative distribution function (cdf), respectively, of $\lambda$. The fading process has been normalized so that $E_{\lambda}[\lambda]=1$. $E_{x}[\cdot]$ denotes the statistical expectation over the random variable $x$. According to the new algorithm, prior to data assignment, subchannels are ordered so that $\lambda_{1} \leq \lambda_{2} \leq \cdots \leq \lambda_{N}$, with $\lambda_{k}=\left|H_{o(k)}\right|^{2}$. The index ordering is taken into account by the function $n=o(k)$. Referring to the order statistic theory results [30], the pdf for $\lambda_{k}$ can be expressed as follows:

$$
\begin{aligned}
f_{k}\left(\lambda_{k}\right) & =\frac{N ! F_{\lambda}\left(\lambda_{k}\right)^{k-1}\left[1-F_{\lambda}\left(\lambda_{k}\right)\right]^{N-k} f_{\lambda}\left(\lambda_{k}\right)}{(k-1) !(N-k) !} \\
& =\frac{N ! e^{-\lambda_{k}(N-k+1)}\left(1-e^{-\lambda_{k}}\right)^{k-1}}{(k-1) !(N-k) !}, \quad k=1, \ldots, N .
\end{aligned}
$$

Looking at (12), we may observe that ordered fading statistics depend on the subchannel index $k$. The average bit error probability on the $k$ th (ordered) subchannel is defined as follows:

$$
\bar{P}_{b_{k}}=\mathrm{E}_{\lambda_{k}}\left[P_{b_{k}}\right],
$$

where the $\mathrm{E}_{\lambda_{k}}\left[P_{b_{k}}\right]$ is obtained by averaging (7) over the statistics given by (12). Considering that only the $N_{u}$ strongest subchannels are used, the final average bit error probability expression becomes

$$
\bar{P}_{b}=\frac{1}{N_{u}} \sum_{k=N-N_{u}+1}^{N} \bar{P}_{b_{k}} .
$$

In [31], it has been verified analytically that the average bit error probability in (14) is minimized for $N_{u}=N / 2$ if $M=4$ (i.e., when only half subchannels and quadruplicated constellation size are used) and $N_{u}=2 N / 3$ if $M=16$. This result shows that the optimum choice of the number of active subchannels $N_{u}$ does not depend on the actual instantaneous SNR but only on the long-term overall channel statistics (in this case, Rayleigh fading). The same minimum has been obtained by simulation in particular practical cases, that is, considering the $5 \mathrm{GHz}$ ETSI channel models [32] and block and convolutional channel coding [31]. The performance gain obtained is induced by the selection of the more reliable subcarriers. 


\subsection{Extension to the multilayer case}

We now extend the considered bit loading algorithm to the multilayer case, where several data streams must be transmitted simultaneously with different performance requirements (UEP) as typical in multimedia applications. In this case, the total number of subchannels is divided into $L$ sets (the number of layers), each one, denoted with $C(l)$, is associated to a different layer. The bit stream, with bit rate $B_{r_{l}}$, associated to each layer is required to have a specific target bit error probability $P_{b_{l}}$. The problem is to find the optimal set of parameters $\left\{C(l), M_{n}, \varepsilon_{n}\right\}(l=1,2, \ldots, L$ and $n=1,2, \ldots, N)$, given $P_{b_{l}}, B_{r_{l}}$, and the channel state $H_{n}$ that minimizes the total transmitted power $P_{T}$. The optimization problem is NPhard [33] and some suboptimal algorithms are present in the literature $[13,24,33]$. They require the knowledge of the relationship between the video quality, in terms of PSNR (see below) or subjective measures, and the correspondent bit error probability $P_{b_{l}}$ required for each layer. However, this relationship is not easy to find as it requires extensive simulation or, alternatively, a model valid in general conditions.

In the paper herein, we investigate a more simple suboptimal scheme capable of realizing UEP at modulation level. It is based on the above-mentioned adaptive ordered subcarrier selection algorithm, where UEP is simply achieved by assigning the bits, belonging to each layer, to subchannels starting from the most reliable down to the least reliable. It must be highlighted that the ordered subcarrier selection algorithm minimizes the overall average bit error probability in (14). However, the layered bit assignment described above leads to an unbalanced average bit error probability between different layers data streams, since bits belonging to more important layers are more protected due to the ordering process.

\section{MPEG-4 CODING}

In order to evaluate the performance of video transmission with the proposed technique, we focused on MPEG-4 [34], the latest MPEG ISO/IEC standard for video compression. The MPEG-4 standard utilizes the concept of object-based coding, allowing interactivity, and layered coding.

The MPEG-4 bitstream is basically structured in video objects (VO's), video object layers (VOL's), that is, the information related to an object in a scalability layer, video $o b$ ject planes (VOP's), that is, the instance of an object in a frame and, optionally, groups of video object planes (GOV's) and packets. Just like most video compression standards, it extensively relies on prediction and entropy coding and it is consequently very sensitive to channel errors.

With the goal of transmission over error prone channels, some error resilience tools have been added to the MPEG-4 standard: reversible variable length codes (RVLC), header extension codes (HEC), resync markers, and data partitioning help in adding robustness to the MPEG-4 bitstream. With the use of resync markers, the MPEG-4 bitstream is composed of packets which are of almost the same length, separated by start codes, unique words recognizable from any sequence of

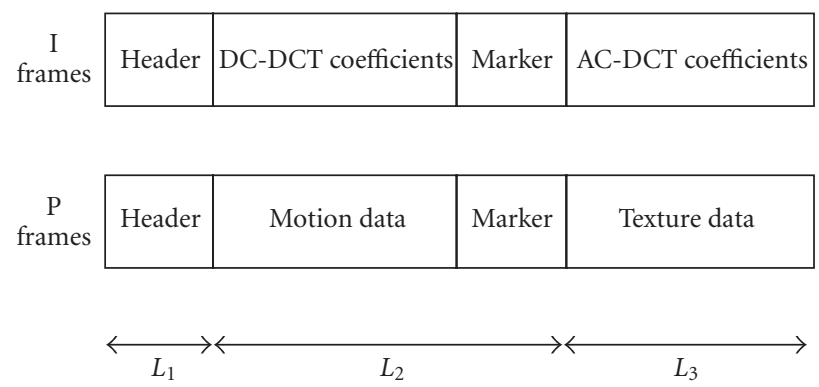

FIGURE 2: Data partitioning of the MPEG-4 packet.

variable length codewords, but not robust to channel errors. The data partitioning tool allows the separation of data with different significances within the packet. Regardless of these tools, MPEG-4 video transmission over wireless channels is still critical: for this reason, studies aimed at efficiently transmitting MPEG-4 video over wireless channels are currently being performed.

If properly exploited, error resilience tools can produce a further improvement of the received video quality. In particular, the data partitioning tool can be usefully exploited with the purpose of performing UEP: information bits contained in each packet are split into three partitions, each of which has a different sensitivity to channel errors.

As shown in Figure 2, for intra (I) frames-reference frames for predictive coding-partitions consist of a header, DC discrete cosine transform (DCT) coefficients, and ACDCT coefficients, separated by a marker. As far as predicted (P) frames are concerned, partitions consist of a header, a motion partition, containing data for the motion compensation, thus very sensitive to channel errors, and a texture partition, separated by a marker. The data partitioning tool may thus be exploited to perform UEP, both at channel coding and at modulation level.

\section{SYSTEM PARAMETERS}

\subsection{Source coding parameters}

In this work, as in [22], we coded according to the MPEG-4 standard the first 13 frames of a video sequence (the "Foreman" test sequence in CIF format) at a bit rate of $644 \mathrm{kbps}$.

The MoMuSys MPEG-4 codec [35] has been used, with some modifications in the decoder, in order to improve the robustness to errors. Additional standard-compatible error resilience techniques have also been adopted. In particular, the lack of robustness in packet/VOP/GOV headers has been afforded with the technique presented in [36], allowing error detection in these critical portions of the bitstream through transparent cyclic redundancy check (CRC); the technique described in [37] has been considered for the reorganization of the bitstream in packets with fixed length and made of fixed length partitions (Figure 3), and for increasing the start codes robustness through substitution with more robust synchronization words. 


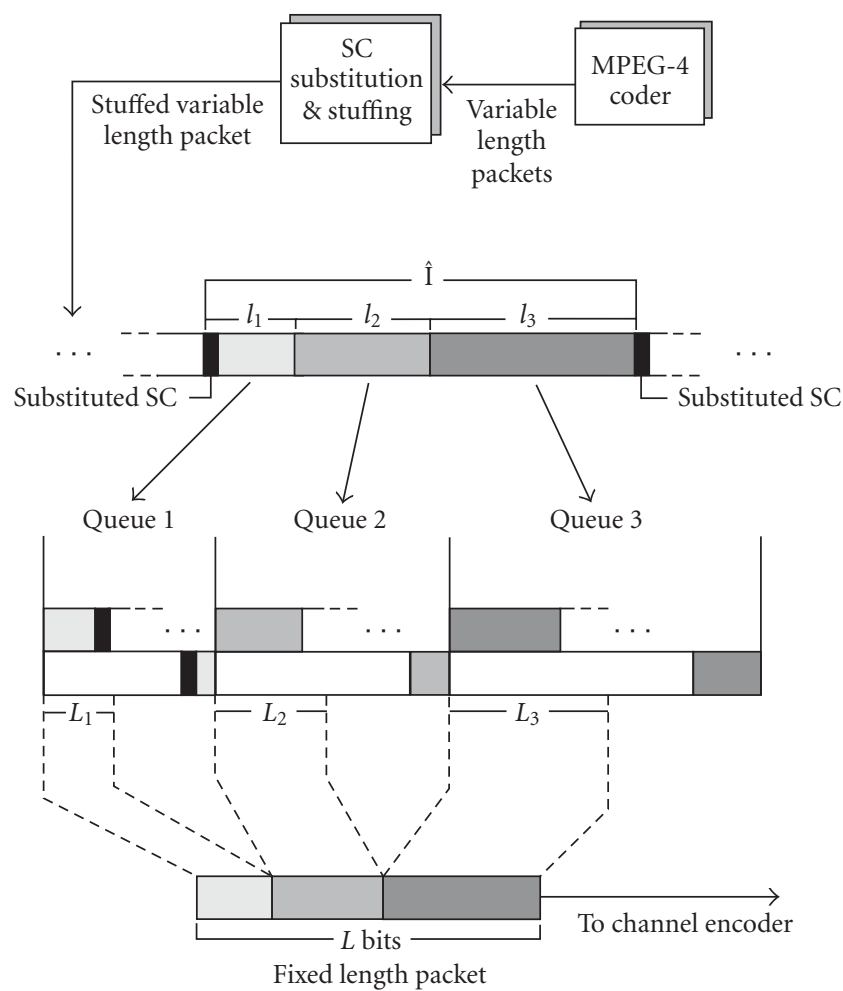

FIgURe 3: Reorganization of the MPEG-4 bitstream in fixed-length packets.

In this work, we have organized the bitstream in packets made of 432 bits, with 27 bits for the first portion of the packet, containing start codes and headers, 108 bits for the second portion of the packet, containing data relevant to the first data partitions, and 297 bits for the last portion, containing data relevant to the second data partitions. Consequently, the unequal protection schemes considered in the paper, both through modulation and through channel codes, refer to a fixed packet structure.

When the UEP is realized at channel coding level, the following coding rates are used for each layer: $R_{c_{1}}=1 / 3$, $R_{c_{2}}=8 / 21, R_{c_{3}}=8 / 13$, for an average code rate of $R_{c} \simeq 1 / 2$. For a fair comparison, when EEP is adopted or UEP is implemented at modulation level, the coding rate is kept constant for all layers to $R_{c}=1 / 2$ as well. Rate compatible punctured recursive systematic convolutional (RCPRSC) codes with rational systematic generator matrix $G s(D)=(1, R 1(D)=$ $\left(1+D+D^{2}+D^{4}\right) /\left(1+D^{3}+D^{4}\right), R 2(D)=\left(1+D^{2}+D^{3}+\right.$ $\left.D^{4}\right) /\left(1+D^{3}+D^{4}\right)$ ), and puncturing period $P=8$ are used [18].

We assume in the following that the first frame is received error free in order to conceal the subsequent frames; we may in fact retransmit the frame if any errors occur since a small delay may be tolerated at the beginning of the bitstream.

\subsection{Transmission system parameters}

Without loss in generality, system parameters are taken from the IEEE802.11a physical layer specifications (or Hiperlan2)
$[1,2]: T_{s}=4$ microseconds, $N_{\mathrm{FFT}}=64, N=48, T_{g}=800$ nanoseconds, subcarrier spacing $\Delta f=312.5 \mathrm{KHz}$. In this case, $\eta_{D}=0.8$.

The total system capacity is kept constant at $b_{T} / T_{s}=$ $24 \mathrm{Mbps}$. Since the average code rate is $R_{c}=1 / 2$, the final useful bit rate becomes $B_{r}=12 \mathrm{Mbps}$. The transmission of one packet requires 10 OFDM frames. As the fixed total bit rate is here $12 \mathrm{Mbps}$, we supposed to send a packet every 678 microseconds, considering others multimedia streams to be transmitted in the remaining time.

As far as the channel model is concerned, we refer to the $5 \mathrm{GHz}$ "E" ETSI channel model [32] (outdoor in non line-ofsight condition) characterized by 18 Rayleigh fading paths. The channel is assumed invariant during the transmission of each packet.

The optimization (bit loading) is performed, according to the temporal evolution of the channel, every $T_{\mathrm{csi}}$ seconds, supposing that the CSI is sent with the same rate. It is advisable that $T_{\mathrm{csi}}<T_{\mathrm{ch}}$, where $T_{\mathrm{ch}}$ is the channel correlation time. In [31], it has been shown that no significant performance degradation is present if $T_{\mathrm{csi}}<7-10$ milliseconds, in the case the user moves with a maximum speed of $3 \mathrm{Km} / \mathrm{h}$.

\section{NUMERICAL RESULTS}

The comparison among the different UEP techniques has been performed for MPEG-4 video transmission over wireless systems, in terms of PSNR. The PSNR is a measure of the video quality defined, in $\mathrm{dB}$, as follows:

$$
\mathrm{PSNR}=20 \log _{10}\left(\frac{255}{\mathrm{RMSE}}\right)
$$

where RMSE is the square root of the mean square error

$$
\mathrm{MSE}=\frac{\sum_{i=1}^{M} \sum_{j=1}^{N}[f(i, j)-F(i, j)]^{2}}{M \times N},
$$

$f(i, j)$ and $F(i, j)$ being the luminance of the pixel $(i, j)$ in the source and the reconstructed images, containing $M \times N$ pixels each. We evaluate the PSNR on the luminance component $(\mathrm{Y})$ of the frame. The PSNR is averaged over the nine $\mathrm{P}$ frames of the first GOV and the first four frames of the second GOV. Results of thirty simulations, performed with different noise seeds, have been averaged in order to obtain more reliable results. The average PSNR is thus

$$
\operatorname{PSNR}_{\text {avg }}=\frac{1}{N_{s} N_{f}} \sum_{s=1}^{N_{s}} \sum_{f=1}^{N_{f}} \operatorname{PSNR}(s, f),
$$

where $N_{s}$ is the number of simulations performed, $N_{f}$ the number of frames considered in the average, and $s$ and $f$ are the simulation index and the frame index. In the case considered, $N_{s}=22$. 
TABLE 1: Schemes considered.

\begin{tabular}{ll}
\hline A & Reference scheme: no adaptation, no UEP. \\
\hline B & $\begin{array}{l}\text { Adaptive ordered subcarrier selection algorithm, } \\
N_{u}=N \text { (UEP through subcarrier reordering). }\end{array}$ \\
\hline C & Adaptive Campello's algorithm, no UEP. \\
\hline D & $\begin{array}{l}\text { Adaptive ordered subcarrier selection algorithm, } \\
N_{u}=N / 2 \text { (UEP through subcarrier reordering). }\end{array}$ \\
\hline E & $\begin{array}{l}\text { Reference scheme: no adaptation, UEP at channel } \\
\text { coding level. }\end{array}$ \\
\hline F & $\begin{array}{l}\text { Adaptive Campello's algorithm, UEP at channel } \\
\text { coding level. }\end{array}$ \\
\hline
\end{tabular}

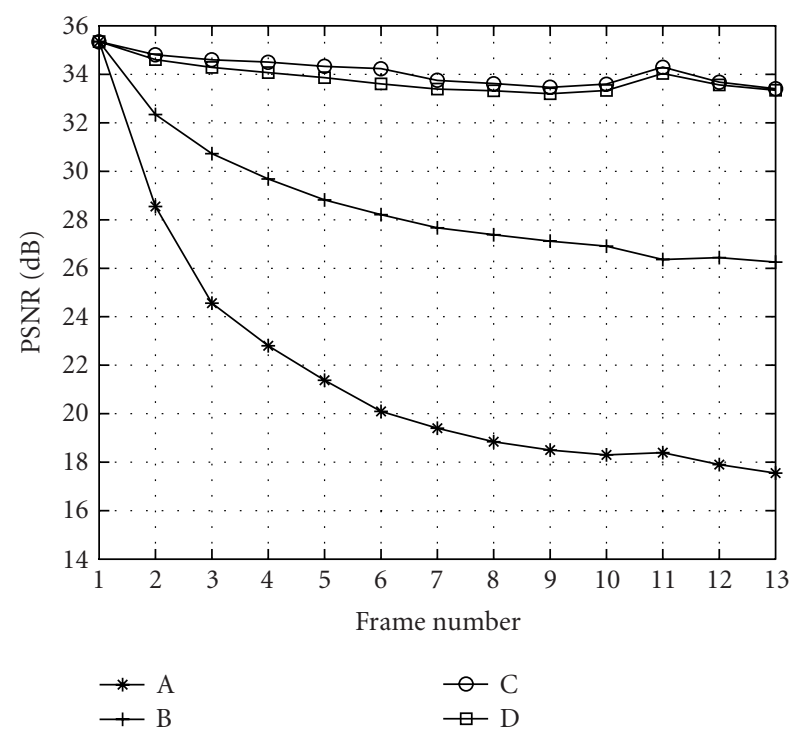

Figure 4: Performance comparison between schemes A, B, C, and $D$ in terms of PSNR $(\mathrm{dB})$ as a function of the frame number for $E_{b} / N_{0}=11 \mathrm{~dB}$ (EEP channel coding).

In the following, we consider the bit loading schemes A, $\mathrm{B}, \mathrm{C}$, and $\mathrm{D}$ with reference to Table 1 .

It is worth noticing that scheme C (Campello) is the optimal bit loading solution but it does not offer the possibility to perform UEP since the bit error rate is the same for all subchannels. On the contrary, the proposed schemes B and $\mathrm{D}$ are suboptimal but they allow the possibility to perform UEP at modulation level due to the subchannel ordering process. Scheme A (no adaptation) is considered for comparison. Also in this case, UEP cannot be performed at modulation level. The different schemes considered are reported for better clarity in Table 1.

In Figures 4 and 5, the evolution of the PSNR as a function of the frame number is shown for schemes A, B, C, and D. The case $E_{b} / N_{0}=11 \mathrm{~dB}$ is reported in Figure 4 where, comparing curves $\mathrm{A}$ and $\mathrm{C}$, it is possible to have an idea of the large gain (up to $18 \mathrm{~dB}$ ) obtainable by the introduction of adaptive loading algorithms. The same gain is achieved with

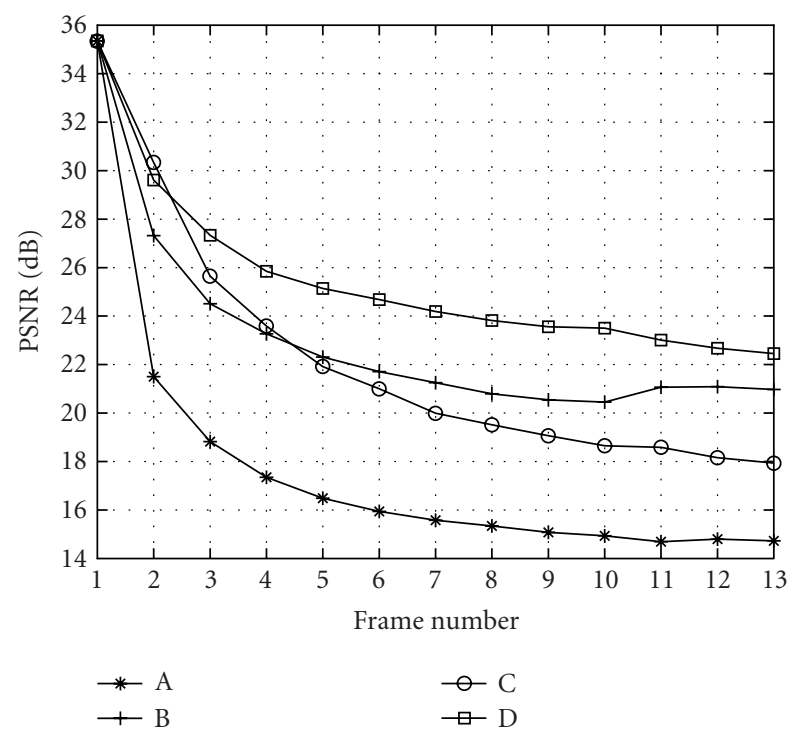

Figure 5: Performance comparison between schemes A, B, C, and $\mathrm{D}$ in terms of PSNR $(\mathrm{dB})$ as a function of the frame number for $E_{b} / N_{0}=7 \mathrm{~dB}$ (EEP channel coding).

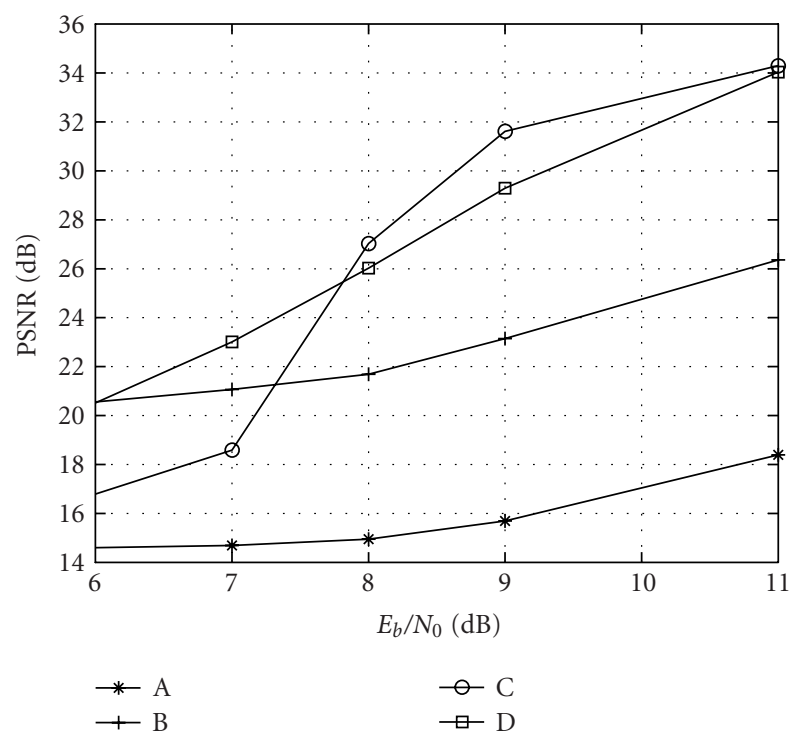

FIGURE 6: PSNR (dB) of frames I versus SNR $\left(E_{b} / N_{0}\right)$ for schemes A, B, C, and D (EEP channel coding).

the simpler scheme $\mathrm{D}$, proposed herein, that employs the UEP at modulation level. The benefit of UEP becomes more evident at lower SNR, as shown in Figure 5 for $E_{b} / N_{0}=7 \mathrm{~dB}$, where both schemes B and D, that implement UEP at modulation level, overcome scheme C. In any case, the gain with respect to the reference scheme (scheme A) remains still remarkable (about $7 \mathrm{~dB}$ ).

The PSNR against $E_{b} / N_{0}$, related to I frames only, is shown in Figure 6. It is interesting to highlight the crossing point between curves $\mathrm{C}$ and D referring to Campello's (EEP) 


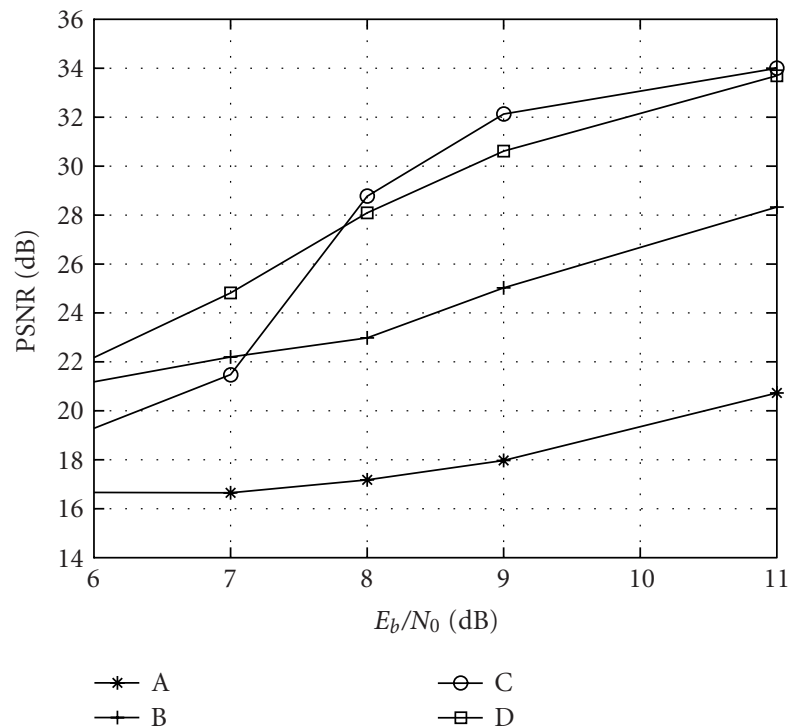

Figure 7: PSNR $(\mathrm{dB})$ of frames P versus SNR $\left(E_{b} / N_{0}\right)$ for schemes A, B, C, and D (EEP channel coding).

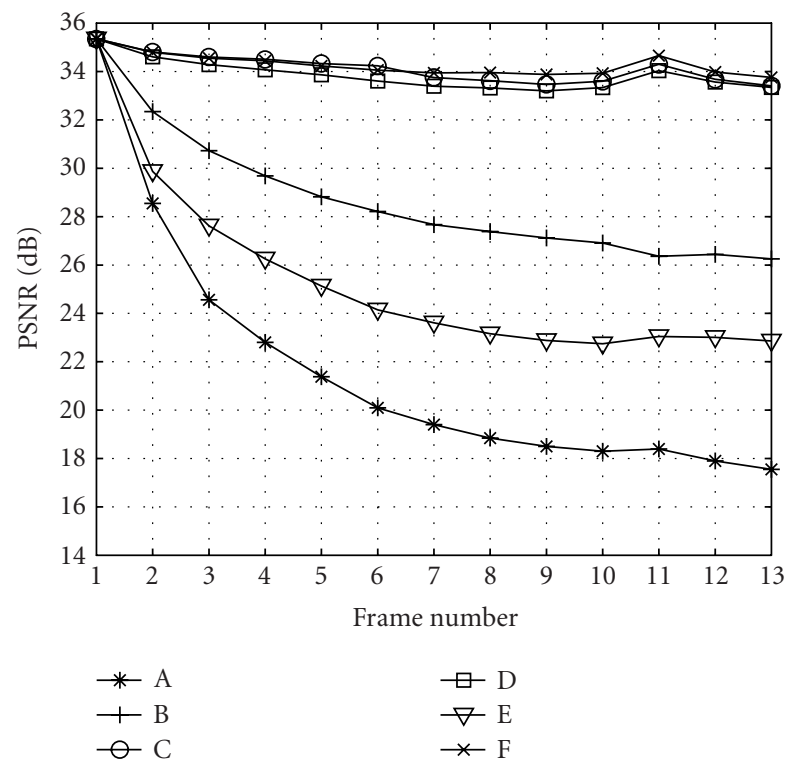

FIgURe 8: Performance comparison between schemes A-F in terms of PSNR $(\mathrm{dB})$ as a function of the frame number for $E_{b} / N_{0}=11 \mathrm{~dB}$. Schemes $\mathrm{E}$ and $\mathrm{F}$ are the same as $\mathrm{A}$ and $\mathrm{C}$ but with UEP realized through channel coding.

and the ordered subcarrier selection algorithms, respectively; at low SNR values, the UEP makes scheme D more robust than scheme C, even though scheme D is simpler and it is suboptimal in single-layer systems. In fact, the gain due to UEP of scheme D at low SNR values is able to compensate for the loss due the suboptimality of bit loading. For better channel conditions (high SNR values), the UEP benefits decreases and cannot compensate for the suboptimality loss of

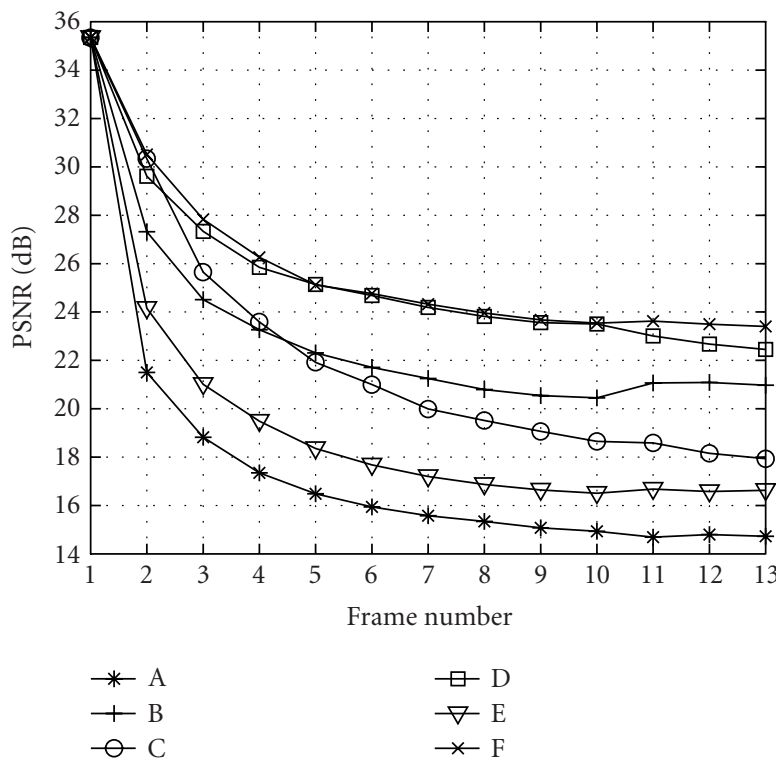

Figure 9: Performance comparison between schemes A-F in terms of PSNR (dB) as a function of the frame number for $E_{b} / N_{0}=7 \mathrm{~dB}$. Schemes $\mathrm{E}$ and $\mathrm{F}$ are the same as $\mathrm{A}$ and $\mathrm{C}$ but with UEP realized through channel coding.

the bit loading algorithm. A similar behavior can be seen in Figure 7 regarding $P$ frames.

The impact of UEP realized through channel coding is illustrated in Figures 8 and 9 for $E_{b} / N_{0}=11 \mathrm{~dB}$ and $E_{b} / N_{0}=$ $7 \mathrm{~dB}$, respectively. Schemes $\mathrm{E}$ and $\mathrm{F}$ are the same as $\mathrm{A}$ and $\mathrm{C}$ but with UEP realized at channel coding level. Schemes A-D are reported for comparison. At high SNR levels (Figure 8), the impact of UEP is significant only when applied to the reference scheme (curve E), but does not give any significant improvement to scheme C (curve F). On the contrary, at lower values for SNR (Figure 9), the performance obtained with scheme $\mathrm{F}$ becomes comparable to the performance of scheme D, where the UEP is realized at modulation level. It should be remarked that scheme $\mathrm{D}$ is much less complex that scheme C.

The visual quality improves too with the adaptive loading technique considered, as shown in Figure 10, where the received frame number 9 of the Foreman sequence is reported for schemes A, C, D, E, and $\mathrm{F}$ in the case of $E_{b} / N_{0}=7 \mathrm{~dB}$. The quality improvement is evident above all for schemes $\mathrm{D}$ and $\mathrm{F}$.

\section{CONCLUSIONS}

In this paper, adaptive loading techniques for multicarrier modulation, applied to Hiperlan2 physical layer system, have been analyzed and compared. A simple multilayer bit loading algorithm has been considered, in order to perform UEP at modulation level, and compared with other bit-loading and UEP schemes. The technique has been applied to MPEG-4 video transmission with good performance gain results over 


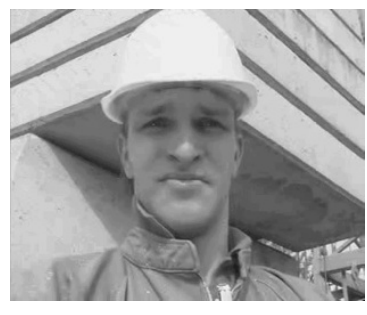

(a) Original.

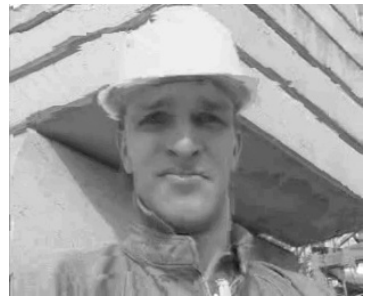

(c) Scheme C.

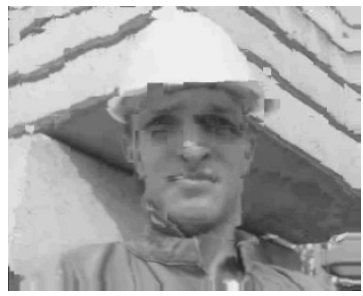

(e) Scheme E.
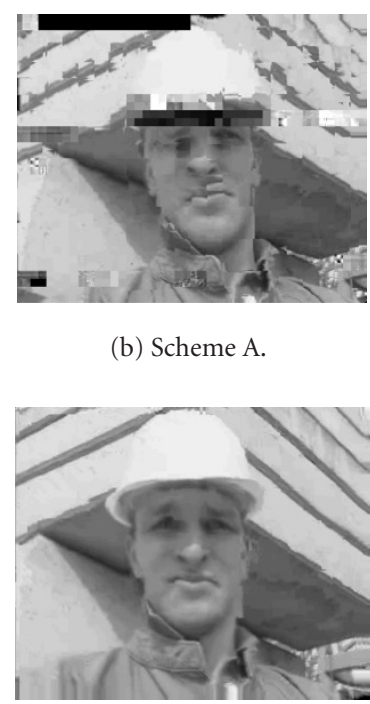

(d) Scheme D.

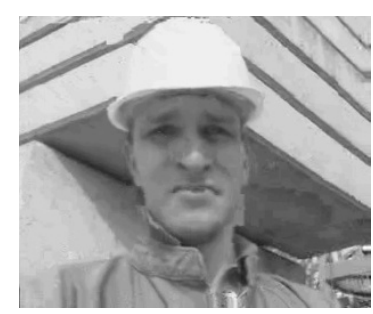

(f) Scheme F. (b) Scheme A.

Figure 10: Frame (P) number 9 of the Foreman sequence. $E_{b} / N_{0}=$ $7 \mathrm{~dB}$.

no adapted schemes, allowing an acceptable video reception also at low SNR values. It has been shown that for high values of SNR, the performance improvement is due mainly to the adaptation of bit loading algorithms to channel impairments, whereas at low SNR values, the advantage introduced by the UEP becomes more significant both at modulation and coding levels.

\section{ACKNOWLEDGMENT}

The work has been partly supported by the Ministero dell'Istruzione, dell'Università e della Ricerca (MIUR) in the framework of the "Virtual Immersive Communication" (VICOM) project.

\section{REFERENCES}

[1] IEEE802.11, part 11, "Wireless LAN Medium Access Control (MAC) and Physical Layer (PHY) Specifications: High Speed Physical Layer in the 5 GHz Band," P802.11a/D7.0, July 1999.

[2] ETSI, "Broadband Radio Access Networks (BRAN) Hiperlan Type2-Physical Layer,” TS 101 475, V 1.1.1, April 2000.

[3] T. Keller and L. Hanzo, "Adaptive multicarrier modulation: a convenient framework for time-frequency processing in wire- less communications," Proceedings of the IEEE, vol. 88, no. 5, pp. 611-640, 2000.

[4] I. Kalet, "The multitone channel," IEEE Trans. Communications, vol. 37, no. 2, pp. 119-124, 1989.

[5] J. A. C. Bingham, ADSL, VDSL, and Multicarrier Modulation, John Wiley \& Sons, New York, NY, USA, 2000.

[6] J. A. C. Bingham, "Multicarrier modulation for data transmission: an idea whose time has come," IEEE Communications Magazine, vol. 28, no. 5, pp. 5-14, 1990.

[7] P. S. Chow, J. M. Cioffi, and J. A. C. Bingham, "A practical discrete multitone transceiver loading algorithm for data transmission over spectrally shaped channels," IEEE Trans. Communications, vol. 43, no. 2/3/4, pp. 773-775, 1995.

[8] R. F. H. Fischer and J. B. Huber, "A new loading algorithm for discrete multitone transmission," in Proc. IEEE Global Telecommunications Conference (GLOBECOM '96), vol. 1, pp. 724-728, London, UK, November 1996.

[9] J. Campello, "Optimal discrete bit loading for multicarrier modulation systems," in Proc. IEEE International Symposium on Information Theory (ISIT '98), p. 193, Cambridge, Mass, USA, August 1998.

[10] A. Czylwik, "Adaptive OFDM for wideband radio channels," in Proc. IEEE Global Telecommunications Conference (GLOBECOM '96), vol. 1, pp. 713-718, London, UK, November 1996.

[11] L. van der Perre, S. Thoen, P. Vandenameele, B. Gyselinckx, and M. Engels, "Adaptive loading strategy for a high speed OFDM-based WLAN," in Proc. IEEE Global Telecommunications Conference (GLOBECOM '98), vol. 4, pp. 1936-1940, Sydney, NSW, Australia, November 1998.

[12] A. N. Barreto and S. Furrer, "Adaptive bit loading for wireless OFDM systems," in Proc. 12th IEEE International Symposium on Personal, Indoor and Mobile Radio Communications (PIMRC '01), vol. 2, pp. G-88-G-92, San Diego, Calif, USA, September-October 2001.

[13] D. Dardari, M. G. Martini, M. Milantoni, and M. Chiani, "MPEG-4 video transmission in the $5 \mathrm{GHz}$ band through an adaptive OFDM wireless scheme," in Proc. 13th IEEE International Symposium on Personal, Indoor and Mobile Radio Communications (PIMRC '02), vol. 4, pp. 1680-1684, Lisboa, Portugal, September 2002.

[14] Q. Su and S. Schwartz, "Effects of imperfect channel information on adaptive loading gain of OFDM," in IEEE 54th Vehicular Technology Conference (VTC '01), vol. 1, pp. 475-478, Atlantic City, NJ, USA, October 2001.

[15] M. R. Souryal and R. L. Pickholtz, "Adaptive modulation with imperfect channel information in OFDM," in Proc. IEEE International Conference on Communications (ICC '01), vol. 6, pp. 1861-1865, Helsinki, Finland, June 2001.

[16] S. Ye, R. S. Blum, and L. J. Cimini Jr., "Adaptive modulation for variable-rate OFDM systems with imperfect channel information," in IEEE 55th Vehicular Technology Conference (VTC '02), vol. 2, pp. 767-771, Birmingham, Ala, USA, May 2002.

[17] W. Xu, S. Heinen, M. Adrat, P. Vary, T. Hindelang, M. Schmautz, and J. Hagenauer, "An adaptive multirate speech codec proposed for the GSM," in Proc. 3rd ITG Conference on Source and Channel Coding, pp. 51-56, Munchen, Germany, January 2000.

[18] J. Hagenauer and T. Stockhammer, "Channel coding and transmission aspects for wireless multimedia," Proceedings of the IEEE, vol. 87, no. 10, pp. 1764-1777, 1999.

[19] A. A. Alatan, M. Zhao, and A. N. Akansu, "Unequal error protection of SPIHT encoded image bit streams," IEEE Journal on Selected Areas in Communications, vol. 18, no. 6, pp. 814-818, 2000. 
[20] H. Gharavi and S. M. Alamouti, "Multipriority video transmission for third-generation wireless communication systems," Proceedings of the IEEE, vol. 87, no. 10, pp. 1751-1763, 1999.

[21] M. Budagavi, W. R. Heinzelman, J. Webb, and R. Talluri, "Wireless MPEG-4 video communication on DSP chips," IEEE Signal Processing Magazine, vol. 17, no. 1, pp. 36-53, 2000.

[22] M. G. Martini and M. Chiani, "Wireless transmission of MPEG-4 video: performance evaluation of unequal error protection over a block fading channel," in Proc. 53rd IEEE Vehicular Technology Conference (VTC'01 Spring), vol. 3, pp. 2056 2060, Rhodes, Greece, May 2001.

[23] ETSI EN 300 744, European Standard, "Digital video broadcasting (DVB): framing structure, channel coding and modulation for digital terrestrial television," 2001.

[24] H. Zheng and K. J. R. Liu, "Robust image and video transmission over spectrally shaped channels using multicarrier modulation," IEEE Trans. Multimedia, vol. 1, no. 1, pp. 88-103, 1999.

[25] S. B. Weinstein and P. M. Ebert, "Data transmission by frequency-division multiplexing using the discrete Fourier transform," IEEE Trans. Communications, vol. 19, no. 5, pp. 628-634, 1971.

[26] D. Dardari and V. Tralli, "High-speed indoor wireless communications at $60 \mathrm{GHz}$ with coded OFDM," IEEE Trans. Communications, vol. 47, no. 11, pp. 1709-1721, 1999.

[27] J. G. Proakis, Digital Communications, McGraw Hill, New York, NY, USA, 4th edition, 2001.

[28] R. Grunheid, E. Bolinth, and H. Rohling, "A blockwise loading algorithm for the adaptive modulation technique in OFDM systems," in IEEE 54th Vehicular Technology Conference (VTC '01), vol. 2, pp. 948-951, Atlantic City, NJ, USA, October 2001.

[29] W. Yu and J. M. Cioffi, "On constant power water-filling," in Proc. IEEE International Conference on Communications (ICC '01), vol. 6, pp. 1665-1669, Helsinki, Finland, June 2001.

[30] H. A. David, Order Statistics, John Wiley \& Sons, New York, NY, USA, 1981.

[31] D. Dardari, "A low complexity and low overhead adaptive bit loading algorithm for OFDM based high-speed WLAN systems," Cost meeting Rep. COST 273 TD(03)155, Prague, Czech Republic, September 2003.

[32] J. Medbo, H. Andersson, and P. Schramm, "Channel models for Hiperlan/2 in different indoor scenarios," Cost meeting Rep. COST 259 TD (98) 070, Bradford, UK, April 1998.

[33] L. M. C. Hoo, J. Tellado, and J. Cioffi, "Discrete dual QoS loading algorithms for multicarrier systems," in Proc. IEEE International Conference on Communications (ICC '99), vol. 2, pp. 796-800, Vancouver, British Columbia, Canada, June 1999.

[34] MPEG-4 standard, MPEG-4 Video Group, "Final Draft of International Standard," ISO-IEC/JTC1/SC29/WG11 N2502a, Atlantic City, NJ, USA, October 1998.

[35] MoMuSys project website, http://www.cordis.lu/infowin/acts/ rus/projects/ac098.htm.

[36] M. G. Martini and M. Chiani, "Joint source-channel error detection with standard compatibility for wireless video transmission," in Proc. IEEE Wireless Communications and Networking Conference (WCNC '02), vol. 1, pp. 215-219, Orlando, Fla, USA, March 2002.

[37] M. G. Martini, M. Mazzotti, and M. Chiani, "Fixed-packetlength transcoding for error resilient video transmission over WCDMA radio links," in Proc. IEEE Packet Video, Nantes, France, April 2003.
D. Dardari was born in Rimini, Italy, on January 19, 1968. He received his Laurea degree in electronic engineering (with the highest honors) and his Ph.D. degree in electronic engineering and computer science from the University of Bologna, Italy, in 1993 and 1998, respectively. In the same year, he joined the Dipartimento di Elettronica, Informatica e Sistemistica to develop his research activity in the area of

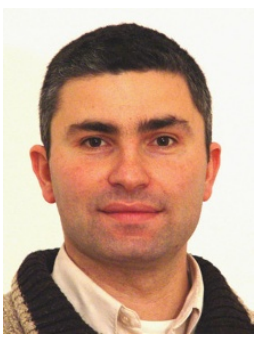
digital communications. During the research activity, he has collaborated and taken a significant role in the following national and European projects: European project "PROMETHEUS" regarding short-range communication systems for cooperative driving; MIUR "WWLAN" project for wideband high-speed wireless LAN; CNIT/ASI (Italian Space Agency) projects "Integration of Multimedia Services on Heterogeneous Satellite Networks" and "Study, Design and Realization of Guaranteed Quality of Service Re-configurable Satellite Network for Multimedia Applications;" MIUR project "VICOM" (Virtual Immersive Communications). Since 2000, he has been a Research Associate at the University of Bologna. He held the position of Lecturer of electrical communications and digital transmission and telecommunications systems at the same university. His research interests are in OFDM systems, nonlinear effects, cellular mobile radio, satellite systems, and wireless LAN. He is a Member and a Reviewer of the IEEE.

M. G. Martini studied electronic engineering at the University of Perugia (Italy) and at the University of Liège (Belgium) and received the Laurea degree in electronic engineering (with the highest honors) from the University of Perugia (Italy) in 1998. After a collaboration with the Aerospace Engineering Department, University of Rome (Italy), she joined in February 1999 the Dipartimento di Elettronica, Informatica e

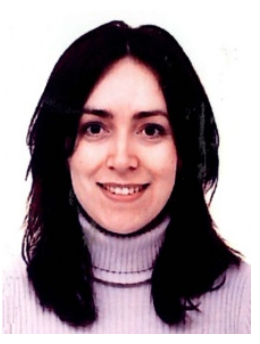
Sistemistica (DEIS), University of Bologna. Here, she has worked as key person for several national and international projects, such as the "Joint Source and Channel Coding" (JSCC) project, in cooperation with Philips Research Monza and Philips Research France, the JOCO (Joint Source and Channel Coding Driven Digital Baseband Design for 4G Multimedia Streaming), and Phoenix (Jointly Optimising Multimedia Transmission in IP Based Wireless Networks) European IST projects. She received the Ph.D. degree in electronics and computer science from the University of Bologna in March 2002. She is currently a Postdoc Researcher at DEIS, University of Bologna. Her research interests are mainly in video coding, channel coding, joint source and channel coding, error resilient video transmission, and wireless multimedia networks.

M. Mazzotti was born in Lugo, Italy, on 12 March 1977. He received the degree in telecommunications engineering (with the highest honors) in July 2002, from the University of Bologna. Now he is working as a Ph.D. student in the Dipartimento di Elettronica, Informatica e Sistemistica in the University of Bologna. His main research interests cover multimedia communications, joint source and channel coding, and wireless communication systems.

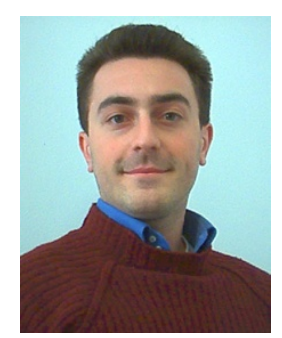


M. Chiani was born in Rimini, Italy, on April 4, 1964. He received the Dr.-Ing. degree (with the highest honors) in electronic engineering and the Ph.D. degree in electronic engineering and computer science from the University of Bologna, Bologna, Italy, in 1989 and 1993, respectively. From 1994, he was with the Dipartimento di Elettronica, Informatica e Sistemistica, University of Bologna, Cesena, where he is cur-

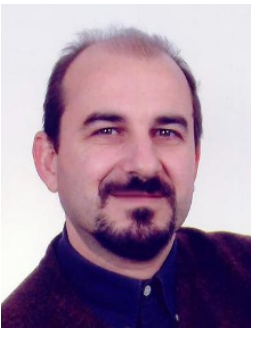
rently a Professor and Chair for telecommunications. His research interests include the areas of communications theory, coding, and wireless networks. Dr. Chiani is an Editor for Wireless Communications, IEEE Transactions on Communications, and Chair of the Radio Communications Committee, IEEE Communications Society. He was in the Technical Program Committees of the IEEE Conferences GLOBECOM 1997, ICC 1999, ICC 2001, ICC 2002, GLOBECOM 2003, and ICC 2004, and is a Senior Member of IEEE. 